\title{
Studying the stereostructures of biomolecules and their analogs by vibrational circular dichroism
}

\author{
Tohru Taniguchi, Takahiro Hongen and Kenji Monde
}

The functions of biomacromolecules are largely governed by their stereostructure (i.e., configuration and conformation). Therefore, detailed understanding of their structural properties should help in regulating the functions of artificial macromolecules. However, studying the stereostructure of a molecule in the solution state is typically difficult due to the lack of suitable analytical techniques. Vibrational circular dichroism (VCD) spectroscopy, which measures circular dichroism in the infrared region, exhibits high sensitivity toward molecular stereostructures. In this paper, we first discuss a method for the elucidation of the stereostructures of small to large molecules based on theoretical calculations of VCD. The rest of the paper is dedicated to the applications of a VCD exciton chirality method, a novel approach recently developed by the authors to interpret VCD data by observing a VCD couplet in the $\mathbf{C}=\mathbf{0}$ stretching region, to various biomolecules such as peptides, carbohydrates, polyesters and lipids.

Polymer Journal (2016) 48, 925-931; doi:10.1038/pj.2016.61; published online 22 June 2016

\section{INTRODUCTION}

Molecular chirality is a fundamental property that governs numerous biological phenomena and is the source of the secondary and higherorder structures of biomolecules. Understanding the chiral structures of nucleic acids, proteins, saccharides and lipids should provide detailed insights into biological systems and help create artificial molecules that mimic or exceed the functions of biomolecules. However, despite extensive studies on chirality, our current level of knowledge does not allow us to thoroughly understand and mimic biomolecular structures, which is partially due to the lack of convenient techniques for analyzing molecular stereostructures. $\mathrm{X}$-ray diffraction analysis has been used to obtain detailed structural data of various molecules, but its efficacy has been limited due to the requirement of a fine crystal. Moreover, this method does not provide structural information in solution. On the other hand, the applicability of solution-state nuclear magnetic resonance spectroscopy is primarily limited to molecules in which hydrogen atoms are suitably located. To understand the details of biological systems, we have been applying vibrational circular dichroism (VCD) spectroscopy ${ }^{1-3}$ to analyze the three-dimensional structures of various biomolecules. For virtually all chiral organic molecules, VCD spectroscopy is capable of detecting signals whose shape reflects their stereostructure. This review describes the methodologies available to analyze the structures of biomolecules and their analogs using VCD spectroscopy, primarily based on our results for peptides, carbohydrates, polyesters and lipids.

\section{METHODOLOGIES FOR STRUCTURAL ANALYSIS USING VCD}

Circular dichroism spectroscopy, which is a measurement of the difference in the absorptions of a chiral sample toward left versus right circularly polarized light, is sensitive to molecular configuration and conformation including subtle structural changes in polymers. Conventional circular dichroism using ultraviolet-visible light, occasionally referred to as electronic circular dichroism (ECD) (Figure 1), has been applied to the conformational analysis of various polymers, such as proteins, nucleic acids, artificial polymers and supramolecules. ${ }^{4}$ However, the applicability of ECD spectroscopy is limited to molecules that contain UV chromophores. VCD is a type of circular dichroism spectroscopy based on infrared radiation and has several advantages over conventional ECD. First, the use of infrared radiation allows for the application of VCD spectroscopy to virtually all chiral organic molecules. Second, VCD can acquire numerous signals that reflect the molecular stereostructure over a wide frequency region. Finally, VCD exhibits superior accuracy for spectral prediction by using theoretical calculations, which leads to the elucidation of detailed molecular structures.

As an example of the determination of the configuration of a small molecule, Figure 2a shows a comparison of the experimentally observed spectra and the theoretically predicted ones using the density functional theory (DFT) calculations of $\alpha$-pinene (1). The observed VCD spectrum of (+)-1 exhibited good agreement with the calculated one for $(1 R, 5 R)-1$ in terms of the peak positions, relative intensities and signs of each signal. Meanwhile, the experimental VCD spectrum for (-)-1 exhibited a pattern that was the mirror image of the theoretical spectrum. These comparisons enable one to elucidate the structures of $(+)-1$ as $1 R, 5 R$ and $(-)-1$ as $1 S, 5 S$.

In contrast to $\mathbf{1}$, most molecules exist in an equilibrated state with more than one conformer. Because of the structural sensitivity and high temporal resolution of VCD spectroscopy (sub-picosecond 
timescale), the observed VCD spectrum is the result of the weighed sum of the contributions from each conformer. Therefore, VCD computations must be carried out for all possible stable conformers of a target molecule and then averaged based on their Boltzmann populations. As a result, the agreement between the obtained theoretical VCD spectrum and the observed one also reveals the conformational nature of the sample. Figure $2 \mathrm{~b}$ shows an example of the conformational elucidation of a simple monosaccharide (2) by VCD. The coupling pattern of $\mathrm{H}-1(\mathrm{dd}, J=3.0,3.0 \mathrm{~Hz})$ indicated that the preferred conformation of 2 in chloroform was ${ }^{1} \mathrm{C}_{4}$, not ${ }^{4} \mathrm{C}_{1}$, because these coupling constants cannot be explained if $\mathrm{H}-1$ was

\begin{tabular}{|c|c|c|}
\hline Wavelength & $200 \mathrm{~nm}$ & $001 \mu \mathrm{m}$ \\
\hline Radiation & Ultraviolet Visible & Infrared \\
\hline $\begin{array}{l}\text { Transition induced } \\
\text { by each radiation }\end{array}$ & Electronic transition & Vibrational transition \\
\hline Corresponding $\mathrm{CD}$ & Conventional $\mathrm{CD}$ & VCD \\
\hline $\begin{array}{l}\text { Absorption of } \\
\text { Organic Molecules }\end{array}$ & $\begin{array}{l}\text { Some molecules with } \\
\text { unsaturated bonds }\end{array}$ & All molecules \\
\hline
\end{tabular}

Figure 1 VCD measures the differential absorption of left versus right circularly polarized light in the infrared (IR) region. Because all organic molecules absorb IR light, VCD is a powerful tool for the analysis of all chiral molecules including nucleic acids, proteins, polyesters and polysaccharides. $C D$, circular dichroism; VCD, vibrational circular dichroism. A full color version of this figure is available at the Polymer Journal journal online. predominantly oriented in an axial position. ${ }^{5}$ Indeed, a molecular mechanics conformation search and subsequent density functional theory optimization of 2 predicted several conformations for both ${ }^{1} \mathrm{C}_{4}$ (conformers 2-(i-iii)) and ${ }^{4} \mathrm{C}_{1}$ (conformers 2-(iv-vi)) with the former being more stable than the latter. The theoretical VCD spectra of each conformer were calculated, as shown in Figure 2b. The spectral patterns of each conformer were significantly different from each other, and none of these patterns were similar to the experimental one. However, the population-average VCD spectrum exhibited good agreement. This result strongly supported the validity of the predicted geometries and the relative stabilities of the conformers of 2 .

In a similar manner, application of VCD theoretical calculations to the homodimer and homotrimer of unnatural amino acids (3a-3d, Figure 2c) allowed us to elucidate their solution-state conformations with non-planar amide linkages. ${ }^{6}$

However, the density functional theory approach to VCD studies of polymers is not directly applicable due to the computational demand of a large molecule, especially when numerous conformers must be taken into account. When the polymer is composed of a single type of monomer (homopolymer), calculations of a truncated model often provide satisfactory structural information as well as the main spectral features of the polymer. ${ }^{7-9}$ Alternatively, when the entire secondary structure of a polymer is uniform, the VCD spectrum of an entire polymer can be simulated by transferring several parameters derived from density functional theory calculations of a small fragment to larger molecules via a Cartesian coordinate transfer technique. $^{10,11}$ However, a computational approach for the a

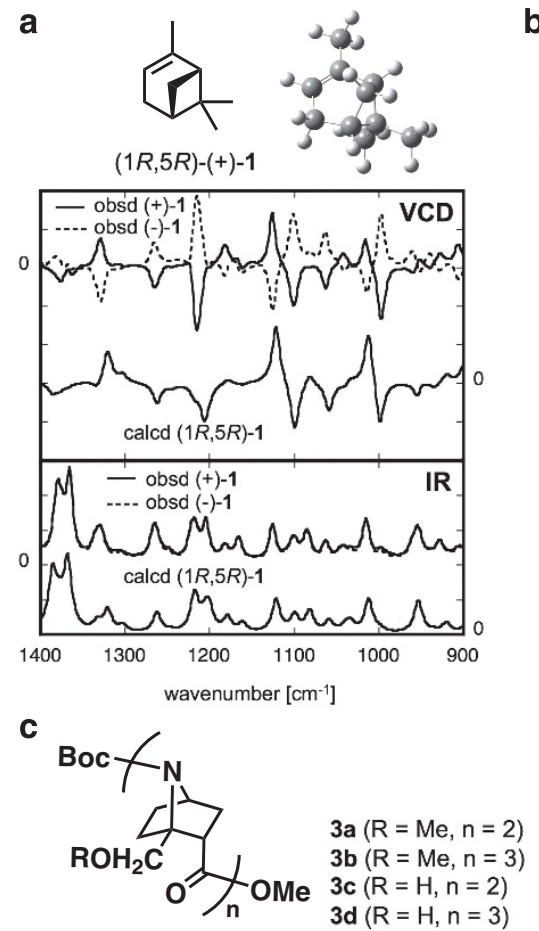

b

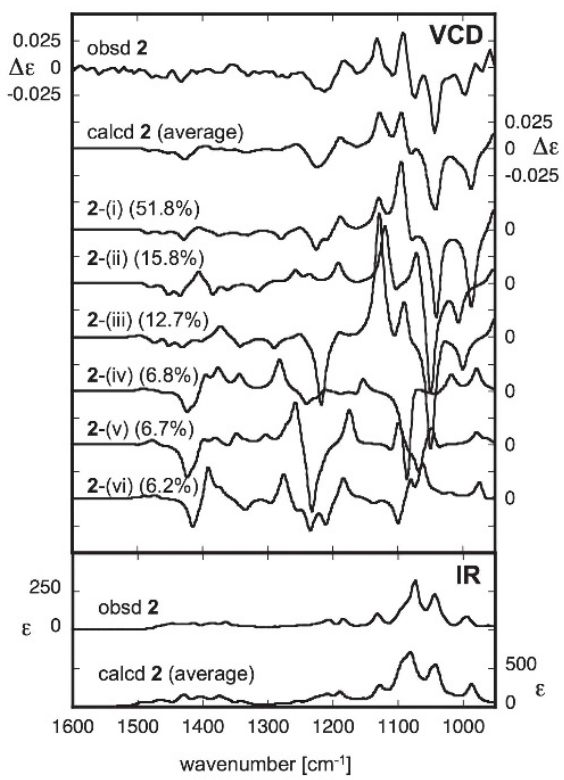

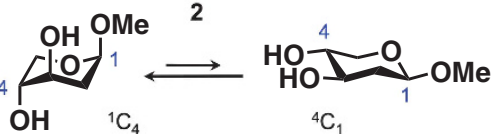

2-(i) $(\Delta E 0.000 \mathrm{kcal} / \mathrm{mol})$

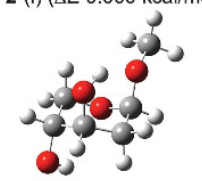

2-(iv) $(\Delta E 1.200 \mathrm{kcal} / \mathrm{mol})$

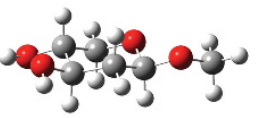

2-(ii) $(\Delta E 0.706 \mathrm{kcal} / \mathrm{mol})$

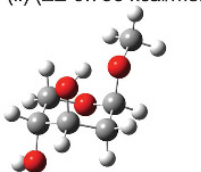

2-(v) $(\Delta E 1.211 \mathrm{kcal} / \mathrm{mol})$

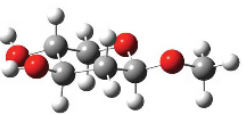

2-(iii) $(\Delta E 0.832 \mathrm{kcal} / \mathrm{mol})$

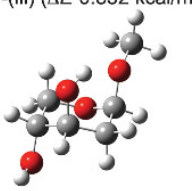

2-(vi) $(\Delta E 1.256 \mathrm{kcal} / \mathrm{mol})$

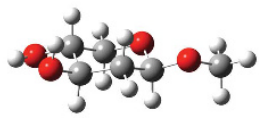

Figure 2 Comparison of the observed and calculated VCD (top) and IR (bottom) spectra of (a) $\alpha$-pinene (1) and (b) methyl 2 -deoxy- $\beta$-D-threo-pentopyranoside (2) as well as (c) the structure of homooligomers of unnatural amino acids (3a-3d). (a) Observed spectra were measured as neat liquid using a JASCO FVS-6000 spectrometer (Tokyo, Japan). Theoretical calculation was conducted for the (1R,5R)-enantiomer at the DFT/B3LYP/6-311G(d,p) level of theory. The only conformer predicted by DFT optimization is also shown. (b) Observed spectra were measured in a $0.1 \mathrm{M} \mathrm{CDCl}_{3}$ solution and corrected by solvent spectra obtained under the same measurement conditions. The theoretical calculations were conducted at the DFT/B3LYP/6-311+G(d,p) level of theory for stable geometries predicted by an Merck molecular force field (MMFF) conformational search. The stable conformers that were taken into account for the VCD and IR calculations are shown along with their relative energies $(\Delta E)$. Their Boltzmann populations were simulated at $298 \mathrm{~K}$. DFT, density functional theory; VCD, vibrational circular dichroism. 
interpretation of the VCD data of polymers with heterogeneous structures must still be developed.

Even without theoretical calculations, VCD spectroscopy has been employed to study the structure of various biomacromolecules in an empirical manner. ${ }^{12}$ In particular, since the early days of VCD spectroscopy, it has been proposed that a pair of carbonyl groups may give rise to a characteristic VCD signal whose shape is indicative of the spatial relationship of the $\mathrm{C}=\mathrm{O}$ groups. ${ }^{13,14}$ Recently, we examined this phenomenon in detail and established a method, which is tentatively referred to as a VCD exciton chirality method (named after the ECD exciton chirality method) ${ }^{15,16}$ to determine the molecular structure without theoretical calculations. ${ }^{17}$ When the absolute twist of the two carbonyl groups is positive $\left(0^{\circ}<\theta<+180^{\circ}\right)$, a positive-negative (from lower to higher frequency) VCD couplet is observed; meanwhile, a negative twist $\left(-180^{\circ}<\theta<0^{\circ}\right)$ yields a negative-positive couplet (Figure 3a). This couplet is due to a

a

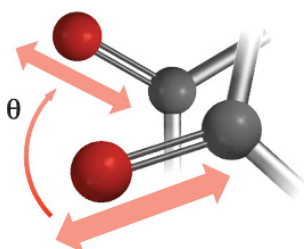

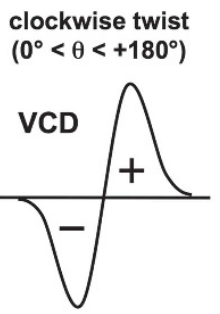

high $\left[\mathrm{cm}^{-1}\right]$ low counterclockwise twist

$\left(-180^{\circ}<\theta<0^{\circ}\right)$

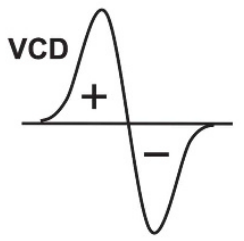

high $\left[\mathrm{cm}^{-1}\right]$ low b
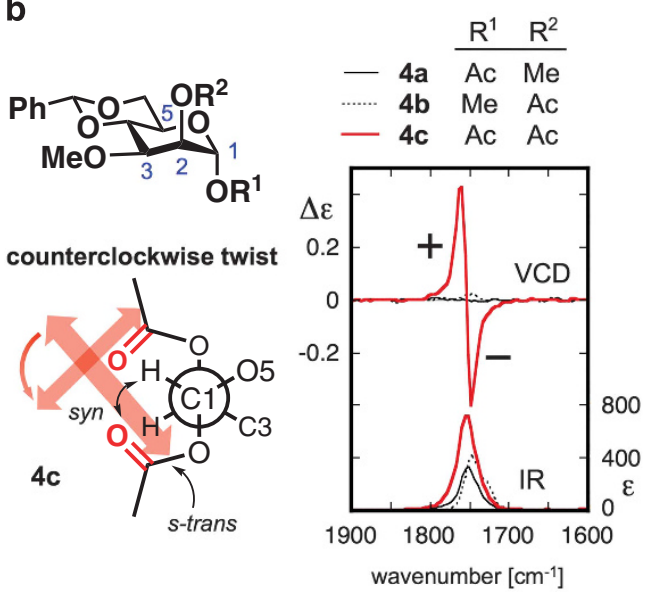

Figure 3 (a) Interaction of two carbonyl groups and the resulting VCD couplet. The sign of the couplet is governed by the torsion angle $(\theta)$ defined by two electric transition moments (red arrows) that are virtually parallel to each $\mathrm{C}=\mathrm{O}$ bond. (b) Comparison of the VCD spectra of mono- and biscarbonyl sugar derivatives $(\mathbf{4 a}-\mathbf{4 c})$. These compounds were measured using $\mathrm{CDCl}_{3}$ at a concentration of $0.05 \mathrm{M}$ and corrected by the solvent spectra obtained under the same measurement conditions. The arrangement of the electric transition moments can be estimated as counterclockwise from the conformational preference of an ester group. VCD, vibrational circular dichroism. through-bond and/or through-space interaction between the two $\mathrm{C}=\mathrm{O}$ chromophores. As shown in Figure 3b, monocarbonyl sugar derivatives $\mathbf{4} \mathbf{a}$ and $\mathbf{4} \mathbf{b}$ exhibited almost flat VCD features in the $\mathrm{C}=\mathrm{O}$ stretching region $\left(1600-1800 \mathrm{~cm}^{-1}\right)$. However, biscarbonyl 4c exhibited an intense VCD couplet whose sign is consistent with the counterclockwise orientation of the two $\mathrm{C}=\mathrm{O}$ groups. The interaction between carbonyl groups becomes weaker as the distance between the two carbonyl groups increases, and therefore, the contribution of two distant carbonyl groups to an observed couplet is often negligible. ${ }^{17,18}$ Accordingly, even if the main chain of a polymer contains numerous carbonyl groups, its observed VCD couplet would be primarily governed by the interactions between pairs of adjacent carbonyl groups. Although the current application of this approach has been limited to a molecule with $\mathrm{C}=\mathrm{O}$ or other functional groups, this method is applicable to large molecules that are beyond the scope of theoretical calculations. In the rest of this paper, we discuss the application of this couplet approach to biomolecules and their analogs. Although this method has proven useful for the structural analysis of small naturally occurring molecules, ${ }^{17-20}$ this topic is not the scope of this review. Similarly, because the aim of this 'Focus Review' is to describe the authors' own research, the details of recent advances in theoretical studies on the VCD couplet phenomenon ${ }^{21,22}$ as well as applications to the structural analysis of films ${ }^{23}$ and gels ${ }^{24,25}$ are not discussed here.

\section{STRUCTURAL ANALYSIS OF GLYCEROPHOSPHOLIPIDS}

Glycerophospholipids (GPLs) are ubiquitous in the membranes of an array of bacterial, archeal and eukaryotic cells. The importance of their amphiphilic nature and structural diversity has been recognized in biology as well as materials science. GPLs are composed of a polar phosphoester headgroup, a common glycerol moiety, and hydrophobic acyl chains with a typical length of $\mathrm{C}_{14}-\mathrm{C}_{20}$. Interestingly, it has been assumed that phosphoesterification of the prochiral glycerol occurs exclusively at the $s n-3$ position for bacterial and eukaryotic GPLs, whereas at the $s n-1$ position for archeal ones, even though no universal method is available to determine their chirality. ${ }^{26}$ However, the presence of a mammalian GPL with $s n-1$ stereochemistry was recently verified, ${ }^{27}$ which suggests the need for a convenient method for analyzing the stereochemistry of GPLs to confirm their previously speculated chirality and elucidate abnormal chirality of other GPLs.

We hypothesized that the two carbonyl groups in GPLs may give rise to a VCD couplet whose sign would reflect their stereochemistry. To test this assumption, several classes of GPLs, such as phosphatidylcholines, phosphatidylserine and bis(monoacylglycero) phosphate containing various lengths of acyl chains with known configuration, were prepared, and their VCD spectra were measured in $\mathrm{CDCl}_{3}$. Despite the expected flexibility of the acyl chains that can fluctuate the orientation of the two $\mathrm{C}=\mathrm{O}$ groups, a clear positive-negative or negative-positive VCD couplet was observed for the measured $s n-3$ or $s n-1$ phosphorylated GPLs, respectively (Figure 4). In agreement with the VCD results, a preliminary conformational calculation also indicated that the two $\mathrm{C}=\mathrm{O}$ groups in an $s n-3$ phosphatidylcholine prefer a clockwise spatial orientation to a counterclockwise one in chloroform. Therefore, these results suggested that the chirality of GPLs can be assigned by simply observing the sign of the $\mathrm{VCD}$ couplet in the $\mathrm{C}=\mathrm{O}$ stretching region. Furthermore, the wide applicability of this assignment method was demonstrated for several GPLs isolated from bacteria, eukaryotes and mitochondria. ${ }^{28}$ 


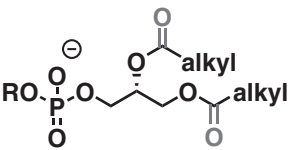

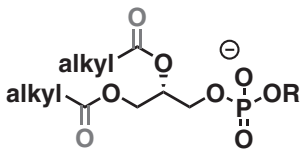

$s n-3$ phosphorylated sn-1 phosphorylated

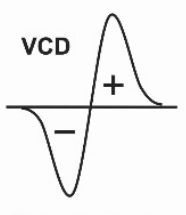

high $\left[\mathrm{cm}^{-1}\right]$ low

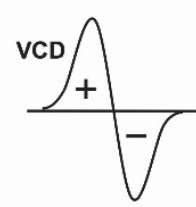

high $\left[\mathrm{cm}^{-1}\right]$ low

Figure 4 Relationship between the stereochemistry of GPL and the sign of the VCD couplet. This relationship is valid for phosphatidylcholines, phosphatidylserine, bis(monoacylglycero)phosphate and cardiolipin irrespective of the length of the acyl chains $\left(\mathrm{C}_{8}-\mathrm{C}_{18}\right)$. GPL, glycerophospholipid; VCD, vibrational circular dichroism. A full color version of this figure is available at the Polymer Journal journal online.

The conformational preference of the two $\mathrm{C}=\mathrm{O}$ groups in GPLs appears to be readily perturbed. For example, VCD measurements of GPL solutions of DMSO- $d_{6}$ and GPL liposomes resulted in the disappearance of the couplet. Although the applicability of this assignment method to other measurement conditions and other classes of GPLs has not been investigated, this VCD analytical method based on the bisignate signal should advance the chemistry and biochemistry of GPLs.

\section{STRUCTURAL ANALYSIS OF PEPTIDES AND PROTEINS}

In a similar manner to the ECD analysis of the secondary structures of polypeptides (for example, $\alpha$-helix, $\beta$-sheet and random coil), ${ }^{29} \mathrm{VCD}$ spectroscopy has long been used as a powerful tool to study their structures. ${ }^{12,30}$ Virtually irrespective of their amino acid sequence, VCD exhibits amide I and II signals characteristic of their secondary structures. The signals in the amide $\mathrm{I}$ region are due to $\mathrm{C}=\mathrm{O}$ stretching vibrations in the amide linkage and the signals in the amide II region originate from $\mathrm{N}-\mathrm{H}$ deformation and other vibrations. Figure $5 \mathrm{a}$ shows a typical example of the VCD spectral features of representative polypeptides measured in $\mathrm{H}_{2} \mathrm{O}$. It is important to note that the spectral shapes are different in $\mathrm{D}_{2} \mathrm{O}$, especially in the amide II region (see detailed review articles). ${ }^{12}$ In $\mathrm{H}_{2} \mathrm{O}$, the right-handed $\alpha$-helix and $3_{10}$ helix typically exhibit a positive-negative couplet in the amide I region and a negative band in the amide II region. In contrast, the left-handed polyproline type II helix exhibits a negative-positive couplet in the amide I region, which is characteristic of the counterclockwise orientation of a pair of adjacent carbonyl groups. Interestingly, the VCD features of random coil closely resemble those of the polyproline type II helix. On the basis of this observation, a random coil has been proposed to contain a large fraction of local lefthanded helices. ${ }^{31} \mathrm{~A} \beta$-sheet structure may yield a negative band in the amide I region, which in some cases is associated with negative and positive signals in the amide II region. Further structural details regarding a specific type of $\beta$-sheet (for example, parallel and antiparallel, in-register and out-of-register, and intersheet stacking patterns) may be obtained using theoretical simulations and/or isotope labeling. ${ }^{32,33}$ When a polypeptide forms a fibril structure, it yields huge bisignate $\mathrm{C}=\mathrm{O}$ stretching $\mathrm{VCD}$ signals whose shapes are completely different from the representative patterns for any known secondary structures. ${ }^{34}$ Although the origin of this enhanced fibril VCD signal is not fully understood, VCD spectroscopy has been able to distinguish macroscopically different fibril supramolecular structures. ${ }^{35,36}$

a

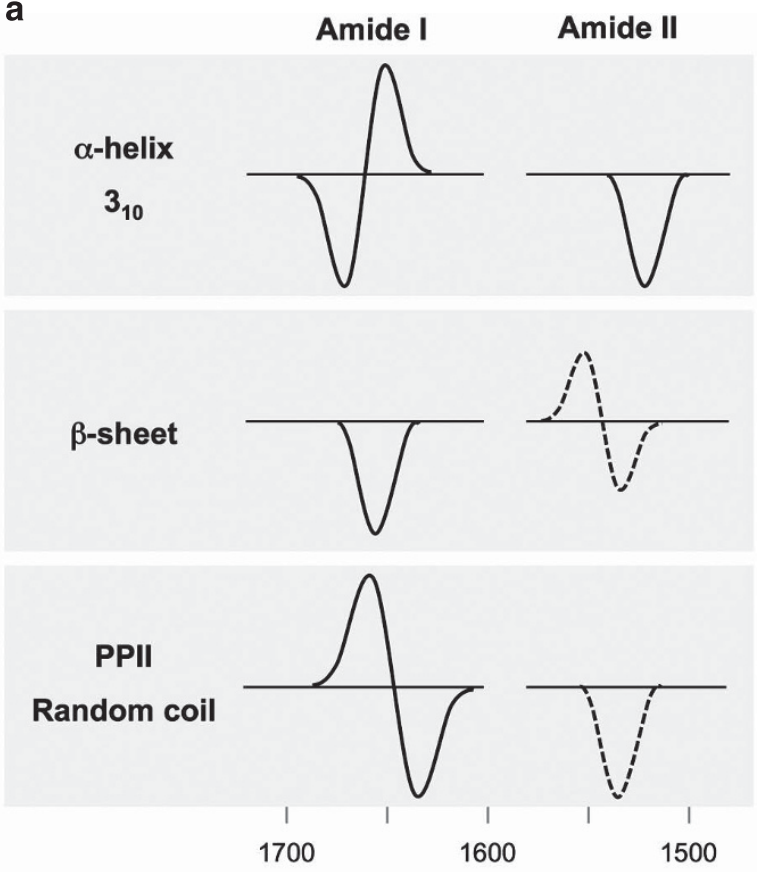

b
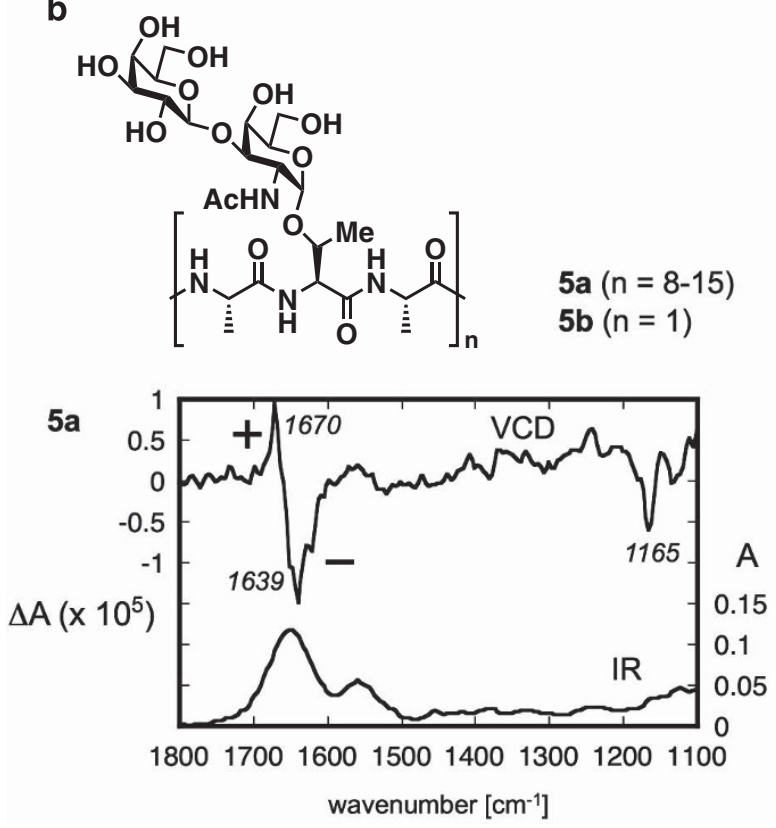

Figure 5 (a) Representative VCD patterns of polypeptides in the amide I and II regions in $\mathrm{H}_{2} \mathrm{O}$. Note that these features can be disturbed depending on the peptide sequence. Different patterns could be observed for spectra recorded in $\mathrm{D}_{2} \mathrm{O}$. (b) $\mathrm{VCD}$ and $\mathrm{IR}$ spectra of glycopeptide antifreeze glycoprotein (AFGP) oligomer (5a) measured as a $500 \mathrm{mg} \mathrm{ml}^{-1} \mathrm{H}_{2} \mathrm{O}$ solution and corrected by the solvent spectra obtained under the same measurement conditions. PPII, polyproline type II; VCD, vibrational circular dichroism. A full color version of this figure is available at the Polymer Journal journal online.

Figure $5 \mathrm{~b}$ shows the IR and VCD spectra of a glycopeptide with antifreeze activity $(\mathbf{5 a})$ measured in $\mathrm{H}_{2} \mathrm{O}$. The disaccharide attached to threonine is essential for the formation of its left-handed polyproline type II helix secondary structure as well as its antifreeze activity. ${ }^{37}$ The VCD spectrum of $\mathbf{5 a}$ contains a negative-positive $\mathrm{C}=\mathrm{O}$ VCD couplet $\left(1639\right.$ and $\left.1670 \mathrm{~cm}^{-1}\right)$, which is indicative of its polyproline 
type II helical structure. A negative VCD band observed at $1165 \mathrm{~cm}^{-1}$ was assigned as vibrational motions that are characteristic of the $\alpha$-glycosidic linkage of $N$-acetylgalactosamine. ${ }^{38}$ The VCD spectrum of tripeptide unit $\mathbf{5 b}$ did not show any strong signals in the $\mathrm{C}=\mathrm{O}$ stretching region, which is consistent with its unordered structure. ${ }^{5,37}$

\section{STRUCTURAL ANALYSIS OF POLYESTERS}

$\operatorname{Poly}(\alpha$-hydroxy acid $)$ is a biodegradable polyester analog of polypeptide. Despite the industrial importance of polyester, its use as a peptidomimetic material has not been fully explored partially due to the difficulties in analyzing and predicting its secondary structure in solution. Unlike polypeptide, many polyesters, such as poly(lactic acid), cannot be studied well using ECD spectroscopy due to their negligibly small UV absorption. ${ }^{39}$ The development of a technique to analyze the secondary structure of polyester will also advance biochemical studies of polypeptides containing $\alpha$-hydroxy acid that can be obtained by a genetic code expansion ${ }^{40,41}$ and chemical synthesis. ${ }^{42}$

Considering the sensitivity of VCD spectroscopy toward the spatial orientation of carbonyl groups, it is reasonable to envision its potential as a tool for studying the secondary structures of polyesters. Nevertheless, application of VCD to polyester had not been reported except for one study by Ho et al. ${ }^{39}$ in 2012 . To establish the usefulness of VCD spectroscopy to polyesters, we applied the VCD couplet approach as well as VCD theoretical calculations to poly(L-lactic acid) (PLLA), PLLA-amylose complex and oligo(L-lactic acid)s (6a-6c); (Figure 6a). ${ }^{43}$ Figure $6 \mathrm{~b}$ shows the VCD spectra of PLLA in $\mathrm{CDCl}_{3}$, PLLA in DMSO- $d_{6}$ and PLLA-amylose complex in DMSO- $d_{6}$. Several conclusions were drawn from these VCD data. (1) A bisignate VCD couplet originating from the $\mathrm{C}=\mathrm{O}$ stretching vibration that is similar to the signals of polypeptide helices was observed in all of the spectra. This observation indicated that VCD spectroscopy is also sensitive to the secondary structure of polyesters. (2) The sign of the couplet (a negative signal at $1751 \mathrm{~cm}^{-1}$ and a positive signal at $1767 \mathrm{~cm}^{-1}$ ) was indicative of a left-handed helix (Figure $6 \mathrm{c}$ ). The left-handedness of PLLA in solution has also been suggested by other researchers. ${ }^{39}$ (3) The VCD spectra of the PLLA solutions of $\mathrm{CDCl}_{3}$ and DMSO- $d_{6}$ were nearly superimposable, suggesting the solvent has little influence on its secondary structure. (4) The VCD signals in the $\mathrm{C}=\mathrm{O}$ stretching region for the PLLA-amylose complex were also virtually superimposable with the other two spectra. Because the shape and intensity of an exciton couplet are governed by the distance and dihedral angle of two chromophores, the close resemblance of these couplets suggests that the conformation of PLLA was mostly maintained even within the cavity inside the amylose helix.

These conclusions were further supported by computational VCD studies of oligo(L-lactic acid)s. For example, the dimer of L-lactic acid (6a), that was predicted to exist as a mixture of left-handed and right-handed helical conformations, exhibited a weak $\mathrm{C}=\mathrm{O}$ couplet. $^{43}$ On the other hand, the tetramer $(\mathbf{6 b})$ and hexamer $(\mathbf{6} \mathbf{c})$ exhibited strong VCD couplets whose intensities are close to that observed for PLLA (Figure 6b). A conformational search and VCD calculation of $6 \mathrm{c}$ indicated that it primarily exists as a left-handed helix in a chloroform solution, which is in agreement with the interpretation of the negative-positive VCD couplet. Therefore, these results not only demonstrated the usefulness of VCD spectroscopy for application to polyesters, but also provided experimental, not just theoretical, evidence of the left-handed property of PLLA in solution. It is important to note that the intrinsic viscosity and light-scattering observations suggested that PLLA was a random coil. ${ }^{44}$ A comparison of these data indicates the possibility that PLLA possesses both 'random coil' and left-handed helical structures (that is, a helix with occasional random coil or a random coil with occasional helix). Another intriguing possibility is that this 'random coil' contains a substantial amount of local left-handed helix similar to a polypeptide 'random coil'. ${ }^{31}$ Although further details are yet to be revealed, VCD spectroscopy will provide experimental insight for unveiling the in-depth structures of polyesters. a<smiles>CC(C)OC(C)C(=O)O</smiles><smiles>COC(=O)C(C)OC</smiles>

PLLA

$6 a(n=2)$

$6 b(n=4)$

$6 c(n=6)$

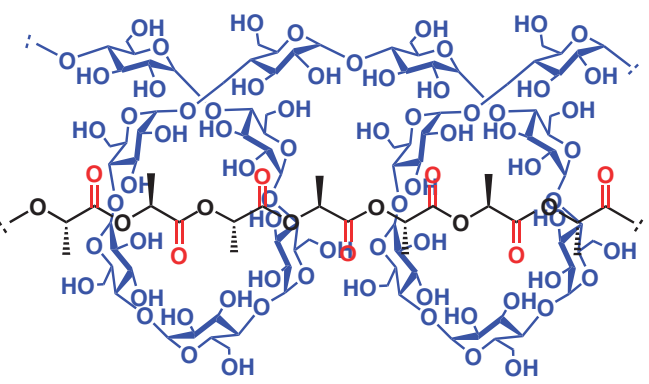

PLLA-amylose complex

b

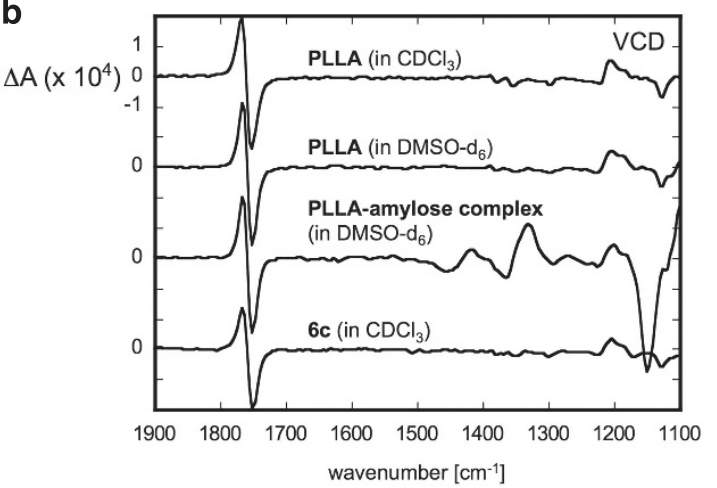

C counterclockwise twist (left-handed helix)

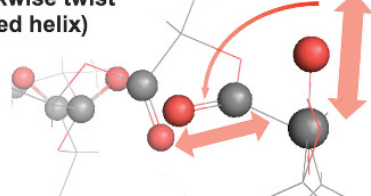

Figure 6 (a) Structures of PLLA, PLLA-amylose complex and oligo(L-lactic acid)s (6a-6c). (b) VCD spectra of PLLA in CDCl 3 (10 mg ml-1), PLLA in DMSO$d_{6}\left(10 \mathrm{mg} \mathrm{ml}^{-1}\right)$, PLLA-amylose complex in DMSO- $d_{6}\left(51 \mathrm{mg} \mathrm{ml}^{-1}\right)$ and $6 \mathrm{c}$ in $\mathrm{CDCl}_{3}\left(8.1 \mathrm{mg} \mathrm{ml}^{-1}\right)$. The spectra of PLLA-amylose and $6 \mathrm{c}$ were normalized based on the area of the $\mathrm{C}=0$ stretching IR band of PLLA in $\mathrm{CDCl}_{3}$. (c) The illustrative arrangement of the carbonyl groups in PLLA drawn based on the most stable conformer predicted for $6 \mathbf{c}$. PLLA, poly(L-lactic acid); VCD, vibrational circular dichroism. 


\section{CONCLUSIONS}

This review discussed the potential of VCD spectroscopy for analyzing the configuration and solution-state conformation of bio(macro) molecules and their analogs. Special emphasis was given to the VCD exciton chirality method, which can be employed to elucidate stereostructures that are difficult to study by other methods. Complementary to classical methods to analyze the global structural properties of macromolecules, the VCD technique provides detailed insights into the local structure with high temporal resolution (sub-picosecond timescale). Further development of the VCD technique will allow for the investigation of various polymers and supramolecules with heterogeneous structures.

\section{CONFLICT OF INTEREST}

The authors declare no conflict of interest.

\section{ACKNOWLEDGEMENTS}

TT acknowledges the financial support from the Japan Society for the Promotion of Science (JSPS) (nos. 23710244 and 26702034), the Shimadzu Science Foundation, the Akiyama Life Science Foundation and the Suhara Memorial Foundation.

1 Nafie, L. A. in Vibrational Optical Activity: Principles and Applications (Wiley, Chichester, UK, 2011)

2 Stephens, P. J., Devlin, F. J. \& Cheeseman, J. R. in VCD Spectroscopy for Organic Chemists (CRC Press, Boca Raton, FL, USA, 2012).

$3 \mathrm{He}$, Y., Wang, B., Dukor, R. K. \& Nafie, L. A. Determination of absolute configuration of chiral molecules using vibrational optical activity: a review. Appl. Spectrosc. 65, 699-723 (2011).

4 Taniguchi, T., Usuki, T. in Supramolecular Chemistry: from Molecules to Nanomaterials, Vol. 2 (eds Gale P. A. \& Steed J. W.), 393-410 (John Wiley \& Sons, Hoboken, NJ, USA, 2012).

5 Taniguchi, T. \& Monde, K. Spectrum-structure relationship in carbohydrate vibrational circular dichroism and its application to glycoconjugates. Chem. Asian J. 2, 1258-1266 (2007)

6 Wang, S., Taniguchi, T., Monde, K., Kawahata, M., Yamaguchi, K., Otani, Y. \& Ohwada, T. Hydrogen bonding to carbonyl oxygen of nitrogen-pyramidalized amide detection of pyramidalization direction preference by vibrational circular dichroism spectroscopy. Chem. Commun. 52, 4018-4021 (2016).

7 Merten, C. \& Hartwig, A. Structural examination of dissolved and solid helical chiral poly (trityl methacrylate) by VCD spectroscopy. Macromolecules 43, 8373-8378 (2010).

8 Tang, H.-Z., Novak, B. M., He, J. \& Polavarapu, P. L. A thermal and solvocontrollable cylindrical nanoshutter based on a single screw-sense helical polyguanidine. Angew. Chem. Int. Ed. Engl. 44, 7298-7301 (2005).

9 Schwartz, E., Liégeois, V., Koepf, M., Bodis, P., Cornelissen, J. J. L. M., Brocorens, P., Beljonne, D., Nolte, R. J. M., Rowan, A. E., Woutersen, S. \& Champagne, B. Beta sheets with a twist: the conformation of helical polyisocyanopeptides determined by using vibrational circular dichroism. Chem. Eur. J. 19, 13168-13174 (2013).

10 Bouř, P., Sopková, J., Bednárová, L., Maloň, P. \& Keiderling, T. A. Transfer of molecular property tensors in cartesian coordinates: a new algorithm for simulation of vibrational spectra. J. Comput. Chem. 18, 646-659 (1997).

11 Andrushchenko, V. \& Bouř, P. Applications of the Cartesian coordinate tensor transfer technique in the simulations of vibrational circular dichroism spectra of oligonucleotides. Chirality 22, E96-E114 (2010).

12 Keiderling, T. A., Lakhani, A. in Comprehensive Chiroptical Spectroscopy, Vol. 2 (eds Berova N., Polavarapu P. L., Nakanishi K. \& Woody R. W.), 707-758 (Wiley, New York, NY, USA, 2012).

13 Holzwarth, G. \& Chabay, I. Optical activity of vibrational transitions: a coupled oscillator model. J. Chem. Phys. 57, 1632-1635 (1972).

14 Freedman, T. B. \& Nafie, L. A. Stereochemical aspects of vibrational optical activity. Top. Stereochem. 17, 113-206 (1987).

15 Harada, N. \& Nakanishi, K. Circular Dichroic Spectroscopy - Exciton Coupling in Organic Stereochemistry (University Science Books, Mill Valley, CA, USA, 1983).

16 Harada, N., Nakanishi, K., Berova, N. in Comprehensive Chiroptical Spectroscopy, Vol. 2 (eds Berova N., Polavarapu P. L., Nakanishi K. \& Woody R. W.), 115-166 (Wiley, New York, NY, USA, 2012).

17 Taniguchi, T. \& Monde, K. Exciton chirality method in vibrational circular dichroism. J. Am. Chem. Soc. 134, 3695-3698 (2012).

18 Asai, T., Taniguchi, T., Yamamoto, T., Monde, K. \& Oshima, Y. Structures of spiroindicumides $\mathrm{A}$ and $\mathrm{B}$, unprecedented carbon skeletal spirolactones, and determination of the absolute configuration by vibrational circular dichroism exciton approach. Org. Lett. 15, 4320-4323 (2013).

19 Komori, K., Taniguchi, T., Mizutani, S., Monde, K., Kuramochi, K. \& Tsubaki, T. Short synthesis of berkeleyamide $D$ and determination of the absolute configuration by the vibrational circular dichroism exciton chirality method. Org. Lett. 16, 1386-1389 (2014).

20 Asai, T., Morita, S., Taniguchi, T., Monde, K. \& Oshima, Y. Epigenetic stimulation of polyketide production in Chaetomium cancroideum by an NAD+-dependent HDAC inhibitor. Org. Biomol. Chem. 14, 646-651 (2016).

21 Abbate, S., Mazzeo, G., Mneghini, S., Longhi, G., Boiadjiev, S. E. \& Lightner, D. A. Bicamphor: a prototypic molecular system to investigate vibrational excitons. J. Phys. Chem. A 119, 4261-4267 (2015).

22 Covington, C. L., Nicu, V. P. \& Polavarapu, P. L. Determination of the absolute configurations using exciton chirality method for vibrational circular dichroism: right answers for the wrong reasons? J. Phys. Chem. A 119, 10589-10601 (2015).

23 Poopari, M. R., Dezhahang, Z., Shen, K., Wang, L., Lowary, T. L. \& Xu, Y. Absolute Configuration and conformation of two Fráter-Seebach alkylation reaction products by film VCD and ECD spectroscopic analyses. J. Org. Chem. 80, 428-437 (2015).

24 Sato, H., Yajima, T. \& Yamagishi, A. Chiroptical studies on supramolecular chirality of molecular aggregates. Chirality 27, 659-666 (2015).

25 Yajima, T., Tabuchi, E., Nogami, E., Yamagichi, A. \& Sato, H. Perfluorinated gelators for solidifying fluorous solvents: effects of chain length and molecular chirality. RSC Adv. $\mathbf{5}$, 80542-80547 (2015).

26 Koga, Y. Early evolution of membrane lipids: how did the lipid divide occur? J. Mol. Evol. 72, 274-282 (2011).

27 Tan, H. H., Makino, A., Sudesh, K., Greimel, P. \& Kobayashi, T. Spectroscopic evidence for the unusual stereochemical configuration of an endosome-specific lipid. Angew. Chem. Int. Ed. Engl. 51, 533-535 (2012).

28 Taniguchi, T., Manai, D., Shibata, M., Itabashi, Y. \& Monde, K. Stereochemical analysis of glycerophospholipids by vibrational circular dichroism. J. Am. Chem. Soc. 137, 12191-12194 (2015).

29 Greenfield, N. J. Methods to estimate the conformation of proteins and polypeptides from circular dichroism data. Anal. Biochem. 235, 1-10 (1996).

30 Keiderling, T. A. Vibrational CD of biopolymers. Nature 322, 851-852 (1986).

31 Dukor, R. K. \& Keiderling, T. A. Reassessment of the random coil conformation: vibrational CD study of proline oligopeptides and related polypeptides. Biopolymers $\mathbf{3 1}$, 1747-1761 (1991).

32 Welch, W. R. W., Kubelka, J. \& Keiderling, T. A. Infrared, vibrational circular dichroism, and Raman spectral simulations for $\beta$-sheet structures with various isotopic labels, interstrand, and stacking arrangements using density functional theory. J. Phys. Chem. B 117, 10343-10358 (2013).

33 Welch, W. R. W., Keiderling, T. A. \& Kubelka, J. Structural analyses of experimental $13 C$ edited amide $I^{\prime} I R$ and VCD for peptide $\beta$-Sheet aggregates and fibrils using DFT-based spectral simulations. J. Phys. Chem. B 117, 10359-10369 (2013).

34 Ma, S., Cao, X., Mak, M., Sadik, A., Walkner, C., Freedman, T. B., Lednev, I. K., Dukor, R. K. \& Nafie, L. A. Vibrational circular dichroism shows unusual sensitivity to protein fibril formation and development in solution. J. Am. Chem. Soc. 129, 12364-12365 (2007).

35 Zhang, G., Babenko, V., Dzwolak, W. \& Keiderling, T. A. Dimethyl sulfoxide induced destabilization and disassembly of various structural variants of insulin fibrils monitored by vibrational circular dichroism. Biochemistry 54, 7193-7202 (2015)

36 Kurouski, D., Lu, X., Popova, L., Wan, W., Shanmugasundaram, M., Stubbs, G., Dukor, R. K., Lednev, I. K. \& Nafie, L. A. Is supramolecular filament chirality the underlying cause of major morphology differences in amyloid fibrils? J. Am. Chem. Soc. 136, 2302-2312 (2014).

37 Tachibana, Y., Fletcher, G. L., Fujitani, N., Tsuda, S., Monde, K. \& Nishimura, S.-I. Antifreeze glycoproteins: elucidation of the structural motifs that are essential for antifreeze activity. Angew. Chem. Int. Ed. Engl. 43, 856-862 (2004).

38 Monde, K., Taniguchi, T., Miura, N. \& Nishimura, S.-I. Specific band observed in VCD predicts the anomeric configuration of carbohydrates. J. Am. Chem. Soc. 126, 9496-9497 (2004)

39 Ho, R.-M., Li, M.-C., Lin, S.-C., Wang, H.-F., Lee, Y.-D., Hasegawa, H. \& Thomas, E. L. Transfer of chirality from molecule to phase in self-assembled chiral block copolymers. J. Am. Chem. Soc. 134, 10974-10986 (2012).

40 Kobayashi, T., Yanagisawa, T., Sakamoto, K. \& Yokoyama, S. Recognition of non- $\alpha$-amino substrates by pyrrolysyl-tRNA synthetase. J. Mol. Biol. 385 , 1352-1360 (2009).

41 Li, Y.-M., Yang, M.-Y., Huang, Y.-C., Li, Y.-T., Chen, P. R. \& Liu, L. Ligation of expressed protein $\alpha$-hydrazides via genetic incorporation of an $\alpha$-hydroxy acid. ACS Chem. Biol. 7, 1015-1022 (2012).

42 Deechongkit, S., Nguyen, H., Powers, E. T., Dawson, P. E., Gruebele, M. \& Kelly, J. W. Context-dependent contributions of backbone hydrogen bonding to $\beta$-sheet folding energetics. Nature 430, 101-105 (2004).

43 Hongen, T., Taniguchi, T., Nomura, S., Kadokawa, J. \& Monde, K. In depth study on solution-state structure of poly(lactic acid) by vibrational circular dichroism. Macromolecules 47, 5313-5319 (2014).

44 Tonelli, A. E. \& Flory, P. J. The configurational statistics of random poly(lactic acid) chains. I. Experimental results. Macromolecules 2, 225-227 (1969). 


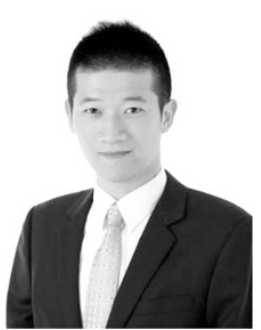

Tohru Taniguchi was born in Tobetsu, Ishikari, Hokkaido in 1980. He received his BS (2002), MS (2004) and PhD (2007) degrees in science from Hokkaido University. During his Master and $\mathrm{PhD}$ courses, he was a YKK scholar (Yoshida Scholarship Foundation, Master 21 and Doctor 21), a JSPS Research Fellow (DC2) and a visiting scholar at Department of Chemistry at Columbia University (20052006). He then became a JSPS Postdoctoral Fellow for Research Abroad and a Uehara Memorial Foundation Postdoctoral Research Fellow during his three-year postdoctoral period at Department of Chemistry at Columbia University, and at Chemistry and Chemical Biology Department at Harvard University (2007-2010). Since 2010, he has been an Assistant Professor at Faculty of Advanced Life Science, Hokkaido University. He has received several awards for his achievement of the development of versatile methods to analyze molecular stereostructures by using chiroptical spectroscopies, including Young Scientist's Research Award in Natural Product Chemistry (Natural Product Chemistry Meeting, 2012), CSJ Award for Young Chemists (Chemical Society of Japan, 2015) and Award for Encouragement of Research in Polymer Science (The Society of Polymer Science, Japan, 2015).

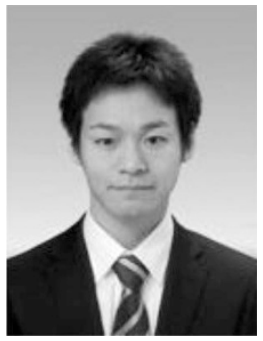

Takahiro Hongen was born in Hokkaido, Japan in 1990 and grew up in Nagano. He received his BS degree in 2013 and MS degree in 2015 from Hokkaido University. He is currently a PhD student at Faculty of Life Science, Hokkaido University. He has been awarded SUNBOR Scholarship from Suntory Foundation for Life Sciences since 2015. His recent research interests are the synthesis of specific oligosaccharides and analysis of supramolecular complexes by VCD.

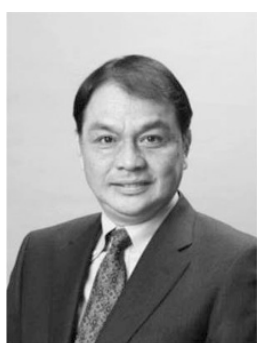

Kenji Monde was born in Hiroshima, Japan in 1961. He received his MS degree from Hokkaido University in 1986 and continued his study on natural product chemistry as a research assistant at the university. After receiving his PhD from Hokkaido University in 1993 , he moved to the Department of Chemistry, Columbia University as a postdoctoral fellow for bioorganic chemistry in 1994 . Then, he moved to Institute for Chemical Reaction Science, Tohoku University, studying chiroptical analysis of biomolecules as an assistant professor in 1996. He returned to Hokkaido University in 2001 as an associate professor. He was promoted to a full professor of Faculty of Advanced Life Science, Hokkaido University in 2010. He has also served as a director of Frontier Research Center for Post-Genome Science and Technology, and a vice dean of Faculty of Advanced Life Science, Hokkaido University since 2013. His recent research interests are chiroptical analysis of biologically active compounds by VCD, discovery of compact molecules that control large biomolecules using new technologies and fluorous chemistry. 